\title{
The effect of birthweight upon insulin resistance and associated cardiovascular risk factors in adolescence is not explained by fetal growth velocity in the third trimester as measured by repeated ultrasound fetometry
}

\author{
S. E. Vielwerth • R. B. Jensen • T. Larsen • K. K. Holst • \\ C. Mølgaard • G. Greisen • A. Vaag
}

Received: 27 May 2007 / Accepted: 18 March 2008 / Published online: 21 May 2008

(C) Springer-Verlag 2008

\begin{abstract}
Aims/hypothesis Smallness for gestational age (SGA) is associated with increased risk of developing components of the metabolic syndrome. Although SGA can imply intrauterine growth restriction (IUGR), more information is required to link specific fetal growth patterns to adult outcomes. Methods We examined the impact of fetal growth velocity during the third trimester (FGV) vs birthweight for gestational age on early markers of the metabolic syndrome
\end{abstract}

S. E. Vielwerth · G. Greisen $(\bowtie)$

University Department of Neonatology, Rigshospitalet,

Section 5023, Blegdamsvej 9,

2100 Copenhagen, Denmark

e-mail: greisen@rh.regionh.dk

R. B. Jensen

University Department of Growth and Reproduction,

Rigshospitalet,

Copenhagen, Denmark

\section{T. Larsen}

Department of Obstetrics and Gynaecology, Sygehus Nord,

Holbaek, Denmark

K. K. Holst

Department of Biostatistics, University of Copenhagen, Copenhagen, Denmark

\section{Mølgaard}

Department of Human Nutrition, Faculty of Life Sciences, University of Copenhagen,

Frederiksberg, Copenhagen, Denmark

\section{A. Vaag $(\bowtie)$}

Steno Diabetes Center,

Niels Stensens Vej 2,

2820 Gentofte, Denmark

e-mail: avaa@steno.dk in 123 healthy men and women (mean age 17.5 years) born at term. FGV was determined by ultrasound measurements. Results After correction for confounders including current BMI, SGA was significantly associated with raised basal plasma insulin $(+19 \%$ above appropriate for gestational age), homeostasis model assessment of insulin resistance $(+21 \%)$, cholesterol:HDL-cholesterol ratio $(+13 \%)$ and systolic BP $(+4.8 \%)($ all $p<0.05)$. Furthermore SGA was associated with increased fat mass $(+9.6 \%)$ and trunk-fat per cent $(+6.8 \%)$ and with reduced lean body mass as determined by dual-energy X-ray absorptiometry scans $(-4.1 \%$ below appropriate for gestational age) (all $p<0.05$ ). In contrast, IUGR in the third trimester was associated only with an elevated cholesterol:HDL-cholesterol ratio $(+11 \%$ above not-IUGR).

Conclusions/interpretation In the present study, FGV did not explain the impact of birthweight upon the metabolic phenotype in adolescence. This suggests that fetal growth prior to the third trimester or postnatal catch-up growth plays a more important role.

Keywords Adiposity - Adolescence - Blood pressure Body composition - Cholesterol - Fetal growth retardation . Fetal weight $\cdot$ Infant $\cdot$ Insulin resistance $\cdot$ Low birthweight

$\begin{array}{ll}\text { Abbreviations } \\ \text { AGA } & \text { appropriate for gestational age } \\ \text { BW } & \text { birthweight } \\ \text { DI } & \text { disposition index } \\ \text { DXA } & \text { dual X-ray absorptiometry } \\ \text { FGV } & \text { fetal growth velocity in the third trimester } \\ \text { FPIR } & \text { first-phase insulin response } \\ \text { HOMA-IR } & \begin{array}{l}\text { homeostasis model assessment of insulin } \\ \text { resistance }\end{array}\end{array}$




$\begin{array}{ll}\text { IUGR } & \text { intrauterine growth restriction } \\ K_{\mathrm{g}} & \text { glucose disappearance rate } \\ \text { SDS } & \text { standard deviation score } \\ \text { SGA } & \text { small for gestational age } \\ \text { SI } & \text { insulin sensitivity }\end{array}$

\section{Introduction}

Studies have indicated that small size at birth is associated with an increased risk of a range of age-related diseases including type 2 diabetes, hypertension, dyslipidaemia and abdominal obesity [1-6]. These metabolic disorders are all related to insulin resistance and the metabolic syndrome, and are associated with an excess cardiovascular mortality. Small size at birth indicates some period(s) of slow fetal growth, and thus poor intrauterine growth conditions have been suggested to be the cause of the observed impact upon later health. However, there also seems to be an interaction between birthweight (BW) and weight gain during infancy and childhood in determining later health [7-10]. Identification of particularly critical periods of fetal life, as well as of individuals at particular risk of later disease, could be crucial for prevention. To acquire evidence for a direct impact of intrauterine growth restriction (IUGR) during specific critical periods, longitudinal data of fetal growth, or data about the intrauterine environment, are required. Previous human studies have explored the effects of maternal nutritional challenges during specific periods of gestation [11, 12]. The Dutch Famine Study found a link between prenatal exposure to famine late in gestation and decreased glucose tolerance later in life [13].

The present study applied a novel approach, trying to pinpoint the critical period(s) of growth in utero. Our a priori hypothesis was that fetal growth velocity in the third trimester (FGV) would contribute significantly to the effect of smallness for gestational age (SGA) upon early markers of the metabolic syndrome. In order to test this hypothesis, we studied adolescent offspring from a unique cohort of mothers, who were previously recruited for ultrasound monitoring of fetal growth during the third trimester based on a priori knowledge of increased risk of delivering an SGA baby.

\section{Methods}

Cohort study of fetal growth (1985-1987) and follow-up (2003-2005)

In a cohort of healthy young men and women, data on gestational age, FGV and BW for gestational age were available from a previous trial of fetometry performed on the mothers in Copenhagen between 1985 and 1987 [14]. In this previous trial, women were recruited if they had one or more risk factors for IUGR or signs of pregnancy complication. One thousand pregnant women were eligible for the trial (Fig. 1). Gestational age was determined by ultrasound at 18 weeks of gestation. Fetal weight was calculated [15] from standardised biometric ultrasound examinations. The test-retest coefficient of variation was $6.5 \%$ and the difference (mean $\pm \mathrm{SD}$ ) between estimated fetal weight and actual BW was $-2.2 \pm 8.7 \%$ [16]. Measurements were performed regularly from week 28 of gestation until delivery, and were transformed to standard deviation scores (SDSs) using a sex-specific reference of fetal growth [16]. One SD corresponds to $11.4 \%$. FGV was determined by linear regression in the 616 individuals with two or more measurements (mean number 4.4) over a period of 42 days or more (mean interval 68 days), and was expressed as $\Delta$ SDS per 28 days. Mean FGV was -0.05 SDS per 28 days ( $\mathrm{SD} \pm 0.38$, range -1.40 to +1.14 ) [17]. The mean error of estimate of the individual FGV in the 610 individuals with three measurements or more was 0.24 SDS per 28 days. The mothers were interviewed about smoking habits at 18 and at 28 weeks of gestation. Use of more than 15 cigarettes a day was considered, and was defined as heavy smoking. BW SDS was calculated using the same growth reference [16]. The correlation between FGV and BW SDS was $0.35(p<0.0001)$ [17]. SGA was defined as BW SDS below the 10th percentile ( $-1.28 \mathrm{SDS})$, conversely appropriate for gestational age (AGA) was a BW SDS above this cut-off. IUGR was defined as declining growth in the third trimester using the cut-off of FGV below the 10th percentile ( -0.39 SDS per 28 days). Five hundred and forty-seven offspring were traceable in 2003 (Fig. 1). All individuals classified as SGA or IUGR were invited to participate in the present follow-up study. Among the individuals classified as AGA/not-IUGR $(n=381), 106$ were randomly selected to participate. One hundred and twenty-three individuals (52 male, 71 female) (mean age $17.5 \pm 0.72$ years) out of 271 agreed to participate. The participants and the group who declined to participate did not differ in BW SDS $(-0.63 \pm 1.21$ vs $-0.61 \pm 1.11$ SDS $)$ $(p=0.88)$ or $\mathrm{FGV}(-0.17 \pm 0.40$ vs $-0.19 \pm 0.38$ SDS per 28 days) $(p=0.78)$. Risk factors for SGA were varied in the studied group. Among these were: maternal heavy smoking (at 18 weeks of pregnancy) $(n=44)$, haemorrhage $(n=41)$, a small uterus, as measured by the distance from the symphysis to the top of the fundus $(n=28)$, previous birth of an SGA child $(n=14)$ and placental insufficiency in a previous pregnancy $(n=11)$. A reference group was examined to assess the potential impact of the generally elevated risk of SGA in our studied cohort. For this purpose, 85 young men and women without data on intrauterine growth were invited to participate; 21 agreed (seven male, 14 
female). They were born by healthy women with no known risk factors for delivering an SGA child, and had a BW SDS between -0.67 SDS and + 0.67 SDS.

Level of physical activity [18], nutrition [19] and smoking habits of the participants and their parents were assessed by questionnaires handed out at follow-up. Furthermore, the parents were asked about breastfeeding (breastfeeding $>3$ months was considered in the present analyses), employment, education and health [20]. Response to the questionnaire was $84 \%$ (participants), $67 \%$ (fathers) and $80 \%$ (mothers). There were no statistical differences in the measured outcomes between the participants who responded to the questionnaire and those who did not.

\section{Experimental protocol}

The participants reported in the morning while fasting. Basal blood samples were drawn. A simplified IVGTT [21] was performed using a glucose bolus (20\%) (300 mg/kg bodyweight) infused over $1 \mathrm{~min}$ and subsequent blood sampling. We measured: waist and hip circumference (using soft tape at the umbilicus and at the widest part of the gluteal region, respectively), skinfold thickness (at biceps, triceps and sub-scapularis using a Harpenden skinfold calliper), height to the nearest $0.1 \mathrm{~cm}$ (using a calibrated wall-mounted stadiometer; Force Institute, Broendby, Denmark) and weight (kg) (using a digital weight scale with a precision of $0.01 \mathrm{~kg}$; Lindeltronic 8000; Copenhagen, Denmark). Fat mass and lean mass were assessed for the whole body as well as for regions of the body using dual x-ray absorptiometry (DXA) measurements performed as whole-body scans (QDR-1000/W [S/N 1108 P] whole-body version 5.61; Hologic, Waltham, MA, USA). Resting BP was measured in a sitting position three consecutive times (digital electronic Omron 705IT blood pressure monitor; Kirudan, Broendby, Denmark).

Female participants who did not use oral contraceptives $(n=29)$ were examined at day 2-5 of their menstrual period. In order to standardise the conditions under which the glucose tolerance test was performed, the participants were instructed to consume a high-carbohydrate diet for 3 days, and to abstain from highly intensive exercise for $24 \mathrm{~h}$ prior to the date of examination.

The study was performed according to the Helsinki II declaration and was approved by the local ethics committee (KF 01-229/02 and KF 01-065/03). Informed consent was obtained from all participants and from the parents when the participant was under 18 years of age.

\section{Biochemical assays}

Plasma insulin blood samples were centrifuged immediately at $4{ }^{\circ} \mathrm{C}$ and stored at $-20^{\circ} \mathrm{C}$ until plasma insulin concentrations were determined by the AutoDELPHIA Time-resolved Fluoroimmunoassay (Perkin Elmer-Wallac

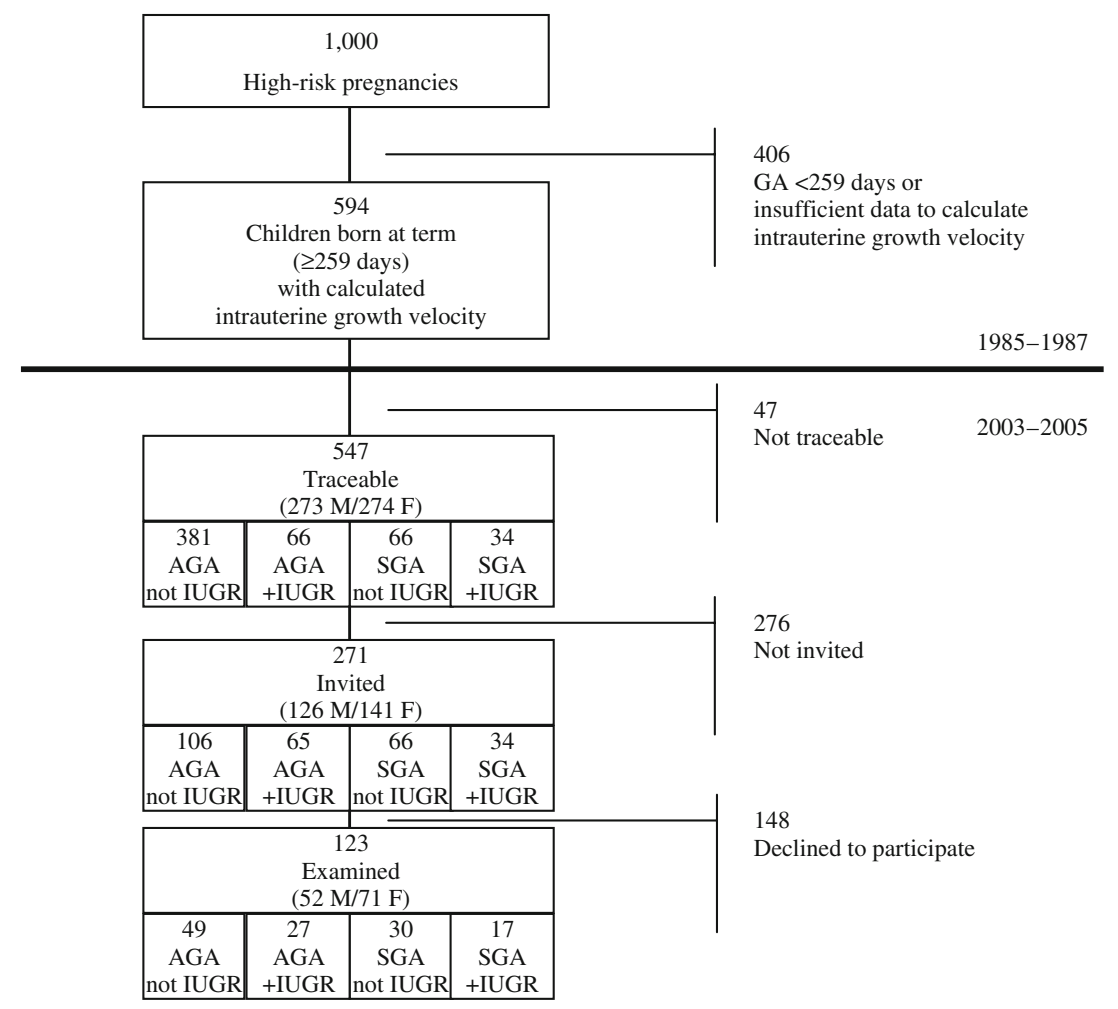

Fig. 1 Study profile. GA, gestational age 
Oy, Turku, Finland) (total interassay $\mathrm{CV}<6 \%$ ). Plasma glucose was measured using the Hexinase/G6P-DH-Determination (Hitachi 912 System; Roche Diagnostics, Hvidovre, Denmark) (intra-assay precision: $\mathrm{SD}=0.09 \mathrm{mmol} / \mathrm{l}$; interassay precision: $\mathrm{SD}=0.15 \mathrm{mmol} / \mathrm{l}) . \mathrm{HbA}_{1 \mathrm{c}}$ was determined using HPLC (G7; TOSOH, Tokyo, Japan) $(\mathrm{CV}<5 \%$ ), and plasma triacylglycerol and cholesterol (total, HDL-cholesterol and LDL-cholesterol) were measured using an enzymatic absorption photometric method (Roche Modular Analytics [SWA] P-module; Roche Diagnostics) $(\mathrm{CV}<5 \%)$.

\section{Calculations}

According to the methods described by Galvin et al. [21] and using the trapezoidal rule we calculated: first-phase insulin response (FPIR) ( $\Delta$ insulin area above $\mathrm{basal}_{0-10 \mathrm{~min}} / \Delta$ glucose peak above basal $_{0-10 \mathrm{~min}}$ ), glucose disappearance rate $\left(K_{\mathrm{g}}\right)\left(-2.303 \times\right.$ slope of $\log$ glucose concentration $\left.{ }_{10-40 \mathrm{~min}}\right)$ and insulin sensitivity (SI) $\left(K_{\mathrm{g}} / \Delta\right.$ insulin area above basal $_{0-40 \text { min }}$ ). Disposition index (DI) representing insulin secretion corrected for the ambient degree of insulin resistance was calculated $\left(\mathrm{SI} \times \Delta\right.$ insulin $\left.\operatorname{area}_{0-10 \min }\right)$. We calculated mean basal glucose (mean of three basal samples), mean basal insulin (mean of three basal samples) and homeostasis model assessment of insulin resistance (HOMA-IR) [22]. Fat per cent SDS was calculated using a national sex-specific chart of fat per cent [23]. Trunk-fat per cent was calculated as trunk-fat mass as a percentage of total fat mass.

\section{Statistical analyses}

In a pre-study power calculation the expected effect size of $0.67 \mathrm{SD}$ (glucose tolerance) corresponded to a required number of participants of 35 with a power of $80 \%$.

All effects of BW SDS, FGV, SGA and IUGR were analysed by multiple regression analyses correcting for sex. Effects of BW SDS and FGV were analysed in common as well as separate analyses. The strategic sampling of all individuals classified as IUGR or SGA and only 106 of the 381 individuals classified as AGA/not-IUGR, entailed a sampling distribution of FGV and BW SDS that was skewed towards lower values. This does not influence the estimated effects of FGV and BW SDS when both of these variables are included in common analyses. But when assessing the effects of FGV and BW SDS separately, we countered this bias by weighting the under-represented group of AGA/not-IUGR by 3.59 (381/106). Furthermore, we calculated the SE of parameters using parametric bootstrap and CIs were calculated from these estimates under normality assumptions. Weighted data and bootstrapped CIs were also applied when the study group was compared with the reference group. The estimated effects derived from weighted regression analyses and 95\% CIs from bootstrapped data are considered be closer to the true effect than effects from unweighted regressions. Unweighted regression analyses generally showed the same tendency and significance of effects for BW SDS as weighted regression analyses.

Effects upon the following parameters were further corrected: glucose-insulin parameters and lipid profile for BMI, body composition for current weight, and BP for BMI, height and age. Age was excluded from all other analyses, because preliminary analyses showed that inclusion did not alter the results. Additional multiple regression analyses of FGV and BW SDS were performed with further correction for each of the following variables in separate analyses: maternal heavy smoking (at week 18 of gestation), fat per cent and trunk-fat per cent. AGA and SGA were compared with regard to answers to the questionnaires using Student's $t$ test for the continuous variables and $\chi^{2}$ test for frequencies. Dependent variables that violated model assumptions were log transformed, which in all analyses gave acceptable model fits. All statistical analyses were performed using the statistical package SPSS (version 14; SPSS, Chicago, IL, USA).

\section{Results}

\section{Volunteer characteristics}

Fetal, birth and follow-up characteristics of the study group are summarised in Table 1 and Table 2. One male individual was excluded from all analyses because of a known diagnosis of type 1 diabetes. Polycystic ovary syndrome had previously been diagnosed in one girl (classified as SGA and IUGR); she was included in the analyses (exclusion did not alter the results).

Effect of risk factors on markers of the metabolic syndrome

The reference group had significantly lower WHR $(B=-0.04$; $95 \% \mathrm{CI}-0.06$ to -0.01 ), and several other measures of adiposity indicated the same tendency. Non-significant trends towards lower basal plasma glucose, higher SI and lower HOMA-IR were also seen in the reference group compared with the study group (data not shown). No other differences were seen between the two groups.

Effects of BW SDS and fetal growth velocity on markers of the metabolic syndrome

Glucose-insulin metabolism BW SDS was significantly and inversely associated with basal insulin and with HOMA-IR after correcting for BMI and sex (Table 3). BW SDS was 
Table 1 Fetal and birth characteristics according to SGA/AGA and IUGR/not-IUGR

\begin{tabular}{|c|c|c|c|c|}
\hline & SGA $(n=47)$ & AGA $(n=75)$ & IUGR $(n=44)$ & Not-IUGR $(n=78)$ \\
\hline \multicolumn{5}{|l|}{ Fetus } \\
\hline Fetal growth velocity (SDS per 28 days) & $-0.25 \pm 0.37$ & $0.03 \pm 0.37$ & $-0.58 \pm 0.17$ & $0.10 \pm 0.30$ \\
\hline \multicolumn{5}{|l|}{ Birth } \\
\hline $\mathrm{BW}(\mathrm{g})$ & $2,724 \pm 302$ & $3,519 \pm 400$ & $3,093 \pm 537$ & $3,427 \pm 465$ \\
\hline BW SDS & $-1.82 \pm 0.48$ & $0.13 \pm 0.87$ & $-0.70 \pm 1.24$ & $-0.14 \pm 1.07$ \\
\hline Gestational age (days) & $278 \pm 9.62$ & $279 \pm 9.02$ & $275 \pm 8.11$ & $279 \pm 9.20$ \\
\hline Birth length $(\mathrm{cm})$ & $48.5 \pm 2.19$ & $51.7 \pm 1.96$ & $49.9 \pm 2.49$ & $51.4 \pm 2.24$ \\
\hline Birth length SDS & $-0.99 \pm 0.69$ & $0.31 \pm 0.72$ & $-0.24 \pm 0.91$ & $0.14 \pm 0.85$ \\
\hline
\end{tabular}

Values are weighted means $\pm \mathrm{SD}$

Table 2 Volunteer characteristics during fetal life, at birth and at adolescence

\begin{tabular}{|c|c|c|c|c|}
\hline & \multicolumn{2}{|c|}{ Female } & \multicolumn{2}{|c|}{ Male } \\
\hline & \multicolumn{2}{|l|}{$n$} & \multicolumn{2}{|l|}{$n$} \\
\hline \multicolumn{5}{|l|}{ Fetus } \\
\hline Fetal growth velocity (SDS per 28 days) & 71 & $-0.18 \pm 0.42$ & 51 & $-0.17 \pm 0.37$ \\
\hline \multicolumn{5}{|l|}{ Birth } \\
\hline BW (g) & 71 & $3,124 \pm 479$ & 51 & $3,287 \pm 580$ \\
\hline BW SDS & 71 & $-0.60 \pm 1.23$ & 51 & $-0.69 \pm 1.19$ \\
\hline Gestational age (days) & 71 & $277 \pm 8.28$ & 51 & $279 \pm 10.2$ \\
\hline Birth length $(\mathrm{cm})$ & 70 & $50.1 \pm 2.44$ & 51 & $50.8 \pm 2.69$ \\
\hline Birth length SDS & 70 & $-0.17 \pm 0.94$ & 51 & $-0.24 \pm 0.95$ \\
\hline \multicolumn{5}{|l|}{ Adolescence } \\
\hline Weight (kg) & 71 & $62.8(55.0-72.6)$ & 51 & $66.5(59.6-76.0)$ \\
\hline Height $(\mathrm{cm})$ & 71 & $167.2(161.5-170.2)$ & 51 & $177.3(172.9-182.5)$ \\
\hline \multicolumn{5}{|l|}{ Glucose-insulin metabolism } \\
\hline Basal glucose $(\mathrm{mmol} / \mathrm{l})$ & 64 & $4.70 \pm 0.34$ & 50 & $5.11 \pm 0.32$ \\
\hline$K_{\mathrm{g}}\left(10^{2} \min ^{-1}\right)$ & 58 & $2.26(1.67-2.87)$ & 47 & $1.66(1.26-2.27)$ \\
\hline Basal insulin (pmol/1) & 67 & $49.5(41.0-62.5)$ & 49 & $35.5(22.1-47.4)$ \\
\hline HOMA-IR & 64 & $1.77(1.32-2.21)$ & 49 & $1.34(0.82-1.91)$ \\
\hline SI $\left(10^{5} \min ^{-1}\left[(\mathrm{pmol} / 1) \times \mathrm{min}^{-1}\right)\right.$ & 58 & $0.32(0.23-0.45)$ & 47 & $0.47(0.30-0.62)$ \\
\hline FPIR $\left([\mathrm{pmol} \times \min ] \mathrm{mmol}^{-1}\right)$ & 58 & $41.8(28.5-61.9)$ & 47 & $26.8(17.7-37.7)$ \\
\hline DI $\left(10^{5} \min ^{-1}\right)$ & 58 & $848(548-1,175)$ & 47 & $673(417-1242)$ \\
\hline \multicolumn{5}{|l|}{ Lipid profile } \\
\hline Triacylglycerol (mmol/l) & 64 & $0.99(0.69-1.31)$ & 47 & $0.69(0.55-0.96)$ \\
\hline Cholesterol $(\mathrm{mmol} / \mathrm{l})$ & 67 & $4.10(3.70-4.70)$ & 51 & $3.50(3.20-4.10)$ \\
\hline LDL-cholesterol (mmol/l) & 67 & $2.40(2.00-3.00)$ & 50 & $2.10(1.68-2.50)$ \\
\hline HDL-cholesterol (mmol/l) & 68 & $1.40(1.20-1.70)$ & 51 & $1.30(1.10-1.50)$ \\
\hline \multicolumn{5}{|l|}{ Body composition } \\
\hline Fat per cent SDS & 67 & $0.97 \pm 1.17$ & 49 & $0.04 \pm 1.12$ \\
\hline Lean body mass (kg) & 67 & $42.4(37.9-46.8)$ & 49 & $53.7(49.2-60.0)$ \\
\hline Trunk fat $(\%)$ & 67 & $36.6 \pm 4.90$ & 49 & $32.1 \pm 5.38$ \\
\hline Fat mass $(\mathrm{kg})$ & 67 & $16.0(14.0-25.0)$ & 49 & $8.23(6.46-10.4)$ \\
\hline WHR & 71 & $0.78(0.74-0.82)$ & 51 & $0.81(0.79-0.85)$ \\
\hline Sum of skinfold thicknesses (mm) & 67 & $37.8(32.2-46.8)$ & 50 & $21.5(18.3-25.5)$ \\
\hline \multicolumn{5}{|l|}{ BP } \\
\hline Systolic (mmHg) & 71 & $118 \pm 9.73$ & 51 & $126 \pm 10.1$ \\
\hline Diastolic (mmHg) & 71 & $71.6 \pm 7.89$ & 51 & $68.8 \pm 7.58$ \\
\hline
\end{tabular}

Values are means $\pm \mathrm{SD}$ or medians (interquartile range) 
Table 3 Effect of BW SDS and FGV upon metabolic and cardiovascular outcome in adolescence

\begin{tabular}{|c|c|c|c|c|}
\hline & \multicolumn{2}{|c|}{ BW SDS } & \multicolumn{2}{|l|}{ FGV } \\
\hline & $B$ & $95 \% \mathrm{CI}$ & $B$ & $95 \% \mathrm{CI}$ \\
\hline \multicolumn{5}{|l|}{ Glucose-insulin metabolism ${ }^{\mathrm{a}}$} \\
\hline Basal glucose $(\mathrm{mmol} / \mathrm{l})$ & -0.02 & -0.07 to 0.03 & -0.09 & -0.31 to 0.14 \\
\hline $\mathrm{HbA}_{1 \mathrm{c}}(\%)$ & -0.03 & -0.07 to 0.02 & -0.08 & -0.24 to 0.08 \\
\hline$K_{\mathrm{g}}\left(10^{2} \min ^{-1}\right)$ & -0.04 & -0.16 to 0.09 & 0.06 & -0.27 to 0.46 \\
\hline Basal insulin (pmol/1) & -2.84 & -4.83 to $-0.74 *$ & -4.82 & -10.3 to 1.65 \\
\hline HOMA-IR & -0.12 & -0.19 to $-0.04 *$ & -0.24 & -0.44 to 0.01 \\
\hline SI $\left(10^{5} \min ^{-1}\left[(\mathrm{pmol} / 1) \times \mathrm{min}^{-1}\right)\right.$ & 0.02 & -0.01 to 0.06 & 0.05 & -0.06 to 0.18 \\
\hline FPIR $\left([\mathrm{pmol} \times \min ] \mathrm{mmol}^{-1}\right)$ & -2.16 & -5.66 to 1.82 & 1.28 & -7.66 to 13.5 \\
\hline DI $\left(10^{5} \min ^{-1}\right)$ & -10.8 & -89.9 to 77.8 & 157 & -81.7 to 481 \\
\hline \multicolumn{5}{|l|}{ Lipid profile $^{\mathrm{a}}$} \\
\hline Triacylglycerol (mmol/l) & -0.07 & -0.14 to $-0.001 *$ & -0.15 & -0.31 to 0.05 \\
\hline Cholesterol (mmol/l) & -0.15 & -0.29 to 0.001 & -0.24 & -0.61 to 0.18 \\
\hline LDL-cholesterol (mmol/l) & -0.14 & -0.26 to $-0.02 *$ & -0.21 & -0.55 to 0.19 \\
\hline HDL-cholesterol (mmol/l) & 0.01 & -0.04 to 0.07 & 0.06 & -0.08 to 0.21 \\
\hline LDL-cholesterol:HDL-cholesterol & -0.12 & -0.21 to $-0.02 *$ & -0.24 & -0.49 to 0.08 \\
\hline Total cholesterol:HDL-cholesterol & -0.14 & -0.22 to $-0.05^{*}$ & -0.28 & -0.55 to 0.02 \\
\hline \multicolumn{5}{|l|}{ Body composition ${ }^{\mathrm{b}}$} \\
\hline Lean body mass $(\mathrm{kg})$ & 0.83 & 0.36 to $1.31^{*}$ & -0.36 & -2.25 to 1.61 \\
\hline Fat mass $(\mathrm{kg})$ & -0.40 & -0.73 to $-0.06^{*}$ & -0.03 & -1.32 to 1.40 \\
\hline Trunk fat $(\%)$ & -1.29 & -1.84 to $-0.74 *$ & -0.47 & -2.68 to 1.74 \\
\hline WHR & -0.01 & -0.02 to 0.00 & -0.02 & -0.04 to 0.01 \\
\hline Sum of skinfold thicknesses (mm) & -0.77 & -1.98 to 0.50 & 1.69 & -2.67 to 6.73 \\
\hline \multicolumn{5}{|l|}{$\mathrm{BP}^{\mathrm{c}}$} \\
\hline Systolic (mmHg) & -1.20 & -3.11 to 0.70 & -1.42 & -5.77 to 2.93 \\
\hline Diastolic (mmHg) & -0.5 & -2.27 to 1.16 & -0.38 & -4.15 to 3.38 \\
\hline
\end{tabular}

Weighted regressions. $B$ coefficients are the effects of BW SDS (units/SDS) and FGV (units/[SDS per 28 days]). BW SDS and FGV analysed separately. $95 \%$ CIs were derived from bootstrapped data

${ }^{a}$ Corrected for BMI and sex

${ }^{\mathrm{b}}$ Corrected for weight and sex

${ }^{\mathrm{c}}$ Corrected for sex, BMI, age, height

${ }^{*} p<0.05$

not associated with glucose tolerance, SI, insulin secretion or DI as determined during IVGTT (Table 3). FGV was not associated with metabolic characteristics in the basal or glucose-stimulated states (Table 3) regardless of correction for BMI or sex.

Lipid profile BW SDS was inversely associated with plasma LDL-cholesterol and triacylglycerol concentrations, as well as with the LDL-cholesterol:HDL-cholesterol and total cholesterol:HDL-cholesterol ratios after correction for BMI and sex (Table 3). FGV and the plasma lipid profile were not associated (Table 3).

Body composition Both BW SDS and FGV were positively associated with current bodyweight corrected for sex $(B=$ $2.01,95 \%$ CI $0.005-4.08 \mathrm{~kg} / \mathrm{SDS}$ and $\mathrm{B}=9.16,95 \% \mathrm{CI}$ $2.70-16.2 \mathrm{~kg} /[\mathrm{SDS}$ per 28 days], respectively). While FGV showed no association with the measured parameters of body composition, BW SDS corrected for current weight was negatively associated with fat mass and trunk-fat per cent and positively associated with lean body mass (Table 3 ).

Blood pressure BW SDS was not associated with diastolic or systolic BP in the weighted regression analyses (Table 3). But in a multiple regression analysis including $\mathrm{FGV}$, systolic but not diastolic BP was inversely associated with BW SDS after correction for BMI, sex, height and age ( $-1.60 \mathrm{mmHg} / \mathrm{SDS} ; 95 \% \mathrm{CI}-3.05$ to $-0.16 \mathrm{mmHg} / \mathrm{SDS})$ $(p=0.030)$. FGV showed no significant association with either diastolic or systolic BP (Table 3).

Multiple regression analyses Multiple regression analyses including both BW SDS and FGV showed that the reported associations between BW SDS and glucose-insulin metabolism, lipid profile (only near significant trends remained) and body composition were independent of FGV, and confirmed the lack of a significant effect of FGV (data not shown). 
Correction for fat per cent, trunk-fat per cent and maternal smoking When BMI was excluded from the multiple regression analyses of $\mathrm{BW}$ SDS and $\mathrm{FGV}$, all the effects of BW SDS were attenuated (for HOMA-IR, $p=0.088$ ). This was also true after correction for fat per cent $(p=0.097$ for HOMA-IR) or trunk-fat per cent ( $p=0.300$ for HOMAIR). Correction for maternal smoking weakened the associations of BW SDS with glucose-insulin metabolism and lipid profile; these became nearly significant $(p=0.053$ for HOMA-IR), but the associations with body composition and systolic BP remained. In this model, maternal heavy smoking was positively associated with trunk-fat per cent in the offspring ( $B=1.46 ; 95 \%$ CI $0.007-2.91 \%)(p=0.049)$, but showed no significant associations with any of other measured parameters in these analyses.

Effects of SGA and IUGR on markers of the metabolic syndrome

Compared with AGA, SGA corrected for confounders as described above was significantly associated with higher basal plasma insulin $(B=19 \% ; 95 \%$ CI 3.4-38) and HOMA-IR ( $B=21 \%$; 95\% CI 3.1-42), higher total cholesterol:HDL-cholesterol ratio $(B=13 \% ; 95 \%$ CI $3.4-23)$, lower lean body mass $(B=-4.1 \% ; 95 \% \mathrm{CI}-6.8$ to -1.4$)$, higher trunk-fat per cent $(B=6.8 \%$; 95\% CI 2.0-11.6) and fat mass $(B=9.6 \% ; 95 \%$ CI 2.6-17), and higher systolic BP ( $B=4.8 \%$; 95\% CI 2.1-7.6) (Fig. 2). Furthermore, nonsignificant trends towards higher triacylglycerol, LDL- cholesterol and total cholesterol were seen for SGA (Fig. 2). IUGR was associated with higher total cholesterol:HDL-cholesterol ratio compared with not-IUGR $(B=$ 11\%; 95\% CI 1.8-20). Other non-significant trends for lipid profile (raised triacylglycerol, LDL-cholesterol and total cholesterol) were seen for IUGR (Fig. 2).

Lifestyle and socioeconomic factors

There were no significant differences between the SGA and AGA group with regard to physical activity, nutrition, breastfeeding and smoking habits of the participants, or employment, education, health, physical activity and nutrition of the parents or the response rate to the questionnaire.

\section{Discussion}

The novelty and uniqueness of the present study relates to the fact that the adolescent study population, because of an a priori increased risk of IUGR, were studied repeatedly with ultrasound measurements of fetal size during the third trimester. This is a potential critical prenatal phase of life, when impaired organ growth and development may result in increased risk of insulin resistance and the metabolic syndrome. We found only little impact of FGV as measured by ultrasound during the third trimester. We observed only one significant effect of declining growth velocity in the third trimester (IUGR), which was a raised total cholesterol:

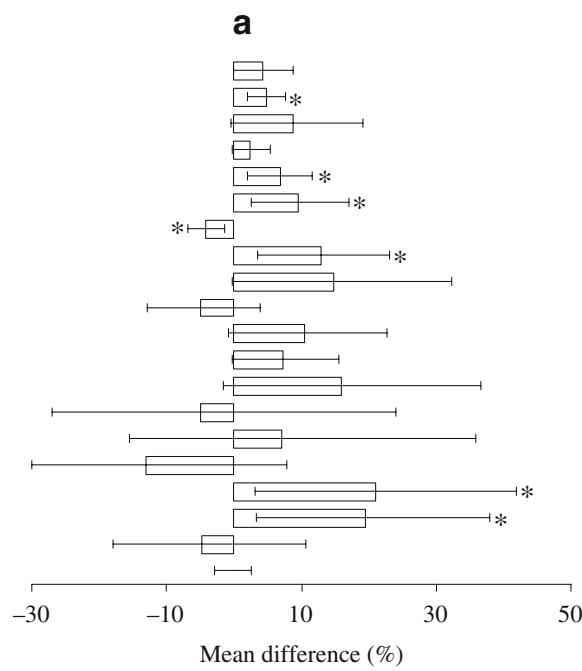

Fig. 2 a The effect of SGA expressed as per cent of AGA. b The effect of IUGR expressed as per cent of not-IUGR. The boxes represent the mean difference and the error bars indicate the $95 \% \mathrm{CI}$ for mean difference as determined by weighted regression analyses. Parameters of glucose-insulin metabolism and lipid profile corrected for BMI and sex. Parameters of body composition corrected for

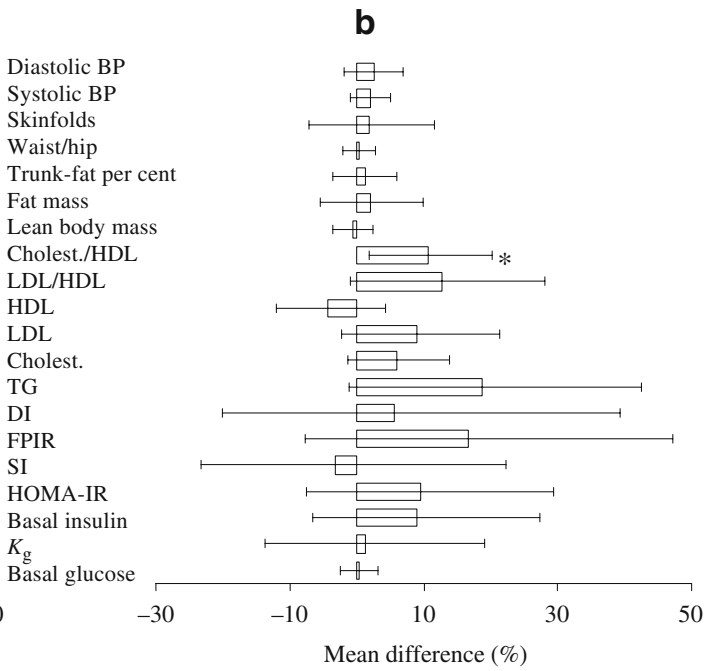

weight and sex. BP corrected for sex, BMI, age and height. CIs derived from bootstrapped data. $* p<0.05$. Values determined from basal blood samples: total cholesterol (Cholest.), HDL-cholesterol (HDL); LDL-cholesterol (LDL), triacylglycerol (TG) and HOMA-IR. Values determined from an IVGTT: DDI, FPIR, SI and $K_{\mathrm{g}}$. Skinfolds, skinfold thicknesses 
HDL-cholesterol ratio. A similar observation was made by Barker et al., who found raised total and LDL-cholesterol in association with low abdominal circumference at birth [24]. A disproportionate effect of growth restriction during the third trimester upon liver growth was proposed as the cause of this association. In our study, however, FGV was not in itself associated with the plasma lipid profile.

In contrast to the potential role of third trimester growth, our results clearly confirm and expand our knowledge of the impact of BW for gestational age. Thus, in our study of an adolescent population, SGA was significantly associated with early markers of virtually all of the key components in the metabolic syndrome. In accord with previous studies [5], we found indications of an association between SGA and insulin resistance. This was determined by HOMA-IR; SI showed the same tendency, although this was not significant. This could be due to a lower power of the analysis, or could alternatively be explained by a tissuespecific increased compensatory glucose uptake, for example in adipose tissue, at higher insulin levels, as suggested by Hermann et al. [25]. Besides early changes in glucoseinsulin metabolism and body composition, we found significant arherogenic changes in the plasma lipid profile and raised BP to be associated with SGA. SGA was not accompanied by diabetogenic changes in insulin secretion, glucose tolerance or DI as seen in other studies [5]. The trend towards raised fasting glucose levels in our young 'at risk' individuals, in comparison to our reference group, might progress to decreased glucose tolerance with increasing age. The observed associations between SGA and lower lean body mass, as well as with higher trunk-fat per cent and fat mass, are in agreement with other studies as reviewed $[4,6]$. Interestingly, when we corrected for fat per cent or trunk-fat per cent instead of BMI, the observed associations between BW SDS and glucose-insulin metabolism, lipid profile and systolic BP were weakened. This finding accentuates the role of (central) adiposity in mediating the effect of low BW, and the necessity for accurate measurements of body composition when exploring these effects.

The number of recruited and eventually studied SGA individuals came close to the number needed according to the power calculation, supporting the validity of the results. In particular, our a priori hypothesis that FGV was more predictive than BW per se for metabolic outcome in adolescence was rejected. The relationships identified in the present study are in accord with previous reports of effects of BW, some of which have shown significant differences between groups of young individuals with low BW compared with control groups of 20 or less each [25-28].

Our comparison of the AGA and SGA group with regard to breastfeeding and socioeconomic and lifestyle factors did not indicate that a difference in these factors was a significant confounder. Maternal heavy smoking seemed to account for a small part of the effect of BW SDS, and to exert its effect through central adiposity, as indicated by the positive association with trunk-fat per cent. Other studies have reported associations of maternal smoking with adiposity in offspring [29-32].

The finding that the effect of BW SDS upon glucoseinsulin metabolism, body composition and BP remained significant in multiple regression analyses including FGV supports the conclusion that it operates independently of third trimester growth. The effect of BW SDS could not be explained by third trimester growth as measured by ultrasound. It is likely, however, that the error of measurement upon fetal weight estimates and thus of FGV was high enough to cause significant 'regression dilution', i.e. to weaken the relationship with metabolic outcomes. Even BW SDS is subject to measurement error from the impression of estimation of gestational age, but this error is likely to be relatively smaller. We did not attempt to correct for these errors in the statistical analysis, since it is unlikely that this could be done well or that this could turn the statistically insignificant results statistically significant. Nevertheless, the conclusions below must be read with caution.

If the effect of SGA is explained by slow growth prior to the third trimester, then the first and second trimesters or perhaps postnatal catch-up growth are the critical windows. In the present study fetal weight SDS at 28 weeks of gestation was negatively associated with trunk-fat per cent in adolescence, but was not associated with other parameters of the metabolic syndrome (data not shown). As programming of central adiposity could be important for the increased risk of poor metabolic and cardiovascular outcomes in SGA individuals, this finding seems to support an important role for growth prior to the third trimester. However, the error of measurement upon a single fetal weight estimate is also relatively high, therefore conclusions based on a single fetal weight estimate should also be drawn cautiously. To reveal the role of earlier periods of growth, the use of longitudinal measurements of fetal size is necessary. In the only (to our knowledge) other published study using this approach, Blake et al. and Gurrin et al. related BP in 6-year-olds to measurement of fetal growth between 18 and 38 weeks of pregnancy, and found an inverse association [33, 34]. We did not find a similar trend regarding third trimester growth (measured from week 28). Although the difference in age of the studied individuals between the two studies may play a role, the difference in results seems to emphasise the assumed importance of fetal growth before the third trimester in determining later BP. Finally, our suggestion regarding early programming (before the third trimester) of the 
significant cardiovascular risk factors of elevated BP, dyslipidaemia and abdominal obesity are consistent with the reports of effects of maternal nutritional restriction in the Dutch Famine Study [35]. However, further research is needed to confirm our findings.

When BMI was excluded from the multiple regression analyses, all the associations between BW SDS and outcome according to metabolic syndrome were attenuated, supporting a central role of obesity in the metabolic syndrome and all of its additional components. Nevertheless, correction for current BMI may simultaneously alter the nature of the exposure $\mathrm{BW}$ to $\mathrm{BW}$ relative to current body size, which in turn, to some unknown extent, points towards the possibility of catch-up growth from birth to current body size as an equally likely explanation (as opposed to growth prior to the third trimester) for the observed associations between BW SDS and metabolic syndrome after corrections for BMI.

The studied group of individuals, born by mothers with an a priori defined increased risk of delivering a SGA child, had a higher index of central obesity and a nearly significant trend towards higher fasting glucose compared with the low-risk reference group. Interestingly, this was independent of $\mathrm{BW}$ and resembles the findings in young low BW men from a normal-risk population as reported in a previous study [26]. In isolation, this novel finding implies a programming effect of a high-risk fetal environment per se independently of fetal growth, and thus in itself supports the hypothesis of developmental origins of adult disease.

\section{Conclusions}

We confirmed the association between SGA and a range of markers of the metabolic syndrome covering insulin resistance, hypertension, dyslipidaemia and abdominal obesity in a young Europid cohort. However, fetal growth velocity during the third trimester, as measured by repeated ultrasound fetometry, did not explain the effect of BW for gestational age. First and second trimester or postnatal catch-up growth may be more critical windows of fetal programming.

Acknowledgements We thank all the participants and parents who took part in the study. We thank J. R. Müller for his efforts in initiating this study. Novo Nordisk, the Lundbeck Foundation, the Danish Health Insurance Foundation, an EXGENESIS grant (005272) from the European Union and the Danish Diabetes Association supported this study. Data from the present study were presented at the 3rd and 4th World Congress on Developmental Origins of Health and Disease in 2005 and 2006, respectively.

Duality of interest The authors declare that there is no duality of interest associated with this manuscript.

\section{References}

1. Huxley R, Owen CG, Whincup PH, Cook DG, Colman S, Collins R (2004) Birth weight and subsequent cholesterol levels: exploration of the 'fetal origins' hypothesis. JAMA 292:2755-2764

2. Huxley RR, Shiell AW, Law CM (2000) The role of size at birth and postnatal catch-up growth in determining systolic blood pressure: a systematic review of the literature. J Hypertens 18:815-831

3. Levy-Marchal C, Jaquet D (2004) Long-term metabolic consequences of being born small for gestational age. Pediatr Diabetes $5: 147-153$

4. McMillen IC, Robinson JS (2005) Developmental origins of the metabolic syndrome: prediction, plasticity, and programming. Physiol Rev 85:571-633

5. Newsome CA, Shiell AW, Fall CH, Phillips DI, Shier R, Law CM (2003) Is birth weight related to later glucose and insulin metabolism? A systematic review. Diabet Med 20:339-348

6. Oken E, Gillman MW (2003) Fetal origins of obesity. Obes Res 11:496-506

7. Weaver LT (2006) Rapid growth in infancy: balancing the interests of the child. J Pediatr Gastroenterol Nutr 43:428-432

8. Metcalfe NB, Monaghan P (2001) Compensation for a bad start: grow now, pay later? Trends Ecol Evol 16:254-260

9. Ong KK (2006) Size at birth, postnatal growth and risk of obesity. Horm Res 65(Suppl 3):65-69

10. Hales CN, Ozanne SE (2003) The dangerous road of catch-up growth. J Physiol 547:5-10

11. Roseboom T, de Rooij S, Painter R (2006) The Dutch famine and its long-term consequences for adult health. Early Hum Dev 82:485-491

12. Stanner SA, Yudkin JS (2001) Fetal programming and the Leningrad Siege study. Twin Res 4:287-292

13. Ravelli AC, van der Meulen JH, Michels RP et al (1998) Glucose tolerance in adults after prenatal exposure to famine. Lancet 351:173-177

14. Larsen T, Larsen JF, Petersen S, Greisen G (1992) Detection of small-for-gestational-age fetuses by ultrasound screening in a high risk population: a randomized controlled study. $\mathrm{Br} \mathrm{J}$ Obstet Gynaecol 99:469-474

15. Warsof SL, Gohari P, Berkowitz RL, Hobbins JC (1977) The estimation of fetal weight by computer-assisted analysis. Am J Obstet Gynecol 128:881-892

16. Larsen T, Petersen S, Greisen G, Larsen JF (1990) Normal fetal growth evaluated by longitudinal ultrasound examinations. Early Hum Dev 24:37-45

17. Larsen T, Greisen G, Petersen S (1997) Intrauterine growth correlation to postnatal growth-influence of risk factors and complications in pregnancy. Early Hum Dev 47:157-165

18. Aadahl M, Jorgensen T (2003) Validation of a new self-report instrument for measuring physical activity. Med Sci Sports Exerc 35:1196-1202

19. Toft U, Kristoffersen LH, Lau C, Borch-Johnsen K, Jorgensen T (2007) The Dietary Quality Score: validation and association with cardiovascular risk factors: the Inter99 study. Eur J Clin Nutr $61: 270-278$

20. Jorgensen T, Borch-Johnsen K, Thomsen TF, Ibsen H, Glumer C, Pisinger C (2003) A randomized non-pharmacological intervention study for prevention of ischaemic heart disease: baseline results Inter99. Eur J Cardiovasc Prev Rehabil 10:377-386

21. Galvin P, Ward G, Walters J et al (1992) A simple method for quantitation of insulin sensitivity and insulin release from an intravenous glucose tolerance test. Diabet Med 9:921-928

22. Matthews DR, Hosker JP, Rudenski AS, Naylor BA, Treacher DF, Turner RC (1985) Homeostasis model assessment: insulin resistance and beta-cell function from fasting plasma glucose and insulin concentrations in man. Diabetologia 28:412-419 
23. Molgaard C, Michaelsen KF (1998) Changes in body composition during growth in healthy school-age children. Appl Radiat Isot 49:577-579

24. Barker DJ, Martyn CN, Osmond C, Hales CN, Fall CH (1993) Growth in utero and serum cholesterol concentrations in adult life. BMJ 307:1524-1527

25. Hermann TS, Rask-Madsen C, Ihlemann N et al (2003) Normal insulin-stimulated endothelial function and impaired insulinstimulated muscle glucose uptake in young adults with low birth weight. J Clin Endocrinol Metab 88:1252-1257

26. Jensen CB, Storgaard H, Dela F, Holst JJ, Madsbad S, Vaag AA (2002) Early differential defects of insulin secretion and action in 19-year-old caucasian men who had low birth weight. Diabetes 51:1271-1280

27. Ozanne SE, Jensen CB, Tingey KJ et al (2006) Decreased protein levels of key insulin signalling molecules in adipose tissue from young men with a low birthweight: potential link to increased risk of diabetes? Diabetologia 49:2993-2999

28. Rasmussen EL, Malis C, Jensen CB et al (2005) Altered fat tissue distribution in young adult men who had low birth weight. Diabetes Care 28:151-153
29. Chen A, Pennell ML, Klebanoff MA, Rogan WJ, Longnecker MP (2006) Maternal smoking during pregnancy in relation to child overweight: follow-up to age 8 years. Int $\mathrm{J}$ Epidemiol 35:121-130

30. Montgomery SM, Ekbom A (2002) Smoking during pregnancy and diabetes mellitus in a British longitudinal birth cohort. BMJ 324:26-27

31. von Kries R, Toschke AM, Koletzko B, Slikker W Jr (2002) Maternal smoking during pregnancy and childhood obesity. Am J Epidemiol 156:954-961

32. Power C, Jefferis BJ (2002) Fetal environment and subsequent obesity: a study of maternal smoking. Int J Epidemiol 31:413-419

33. Blake KV, Gurrin LC, Beilin LJ et al (2002) Prenatal ultrasound biometry related to subsequent blood pressure in childhood. J Epidemiol Community Health 56:713-718

34. Gurrin LC, Blake KV, Evans SF, Newnham JP (2001) Statistical measures of foetal growth using linear mixed models applied to the foetal origins hypothesis. Stat Med 20:3391-3409

35. Painter RC, Roseboom TJ, Bleker OP (2005) Prenatal exposure to the Dutch famine and disease in later life: an overview. Reprod Toxicol 20:345-352 